\title{
Tailoring Particle Size and Agglomeration State of Mesoporous MCM-48 via Optimisation of Sol-gel Silica Process
}

\author{
Nurul Afiqah Mokri, Oh Pei Ching, ${ }^{*}$ Hilmi Mukhtar and Chew Thiam Leng \\ $\mathrm{CO}_{2}$ Research Centre (CO2RES), Institute of Contaminant Management, \\ Department of Chemical Engineering, Universiti Teknologi PETRONAS, \\ 32610 Seri Iskandar, Perak, Malaysia
}

*Corresponding author: peiching.oh@utp.edu.my

Published online: 25 April 2019

To cite this article: Mokri, N. A. et al. (2019). Tailoring particle size and agglomeration state of mesoporous MCM-48 via optimisation of sol-gel silica process. J. Phys. Sci., 30(1), 145-168, https://doi.org/10.21315/jps2019.30.1.11

To link to this article: https://doi.org/10.21315/jps2019.30.1.11

\begin{abstract}
Mobil Composition of Matter no. 48 (MCM-48) is a promising inorganic particle due to its unique crystal structure and 3-dimensional porous network. Incorporating a discrete amount of nanosized MCM-48 for material development would give significant impact to the material's performance and mechanical robustness. Thus far, the synthesis of MCM-48 in the nanoscale regime has been achieved via chemical modification techniques. However, they are often not reproducible due to limited understanding of surfactant behaviour. On the other hand, physical modification technique is more stable, thereby can reduce the possibility of producing other undesired mesophase. Nevertheless, this technique has not been adapted for nanosized MCM-48 synthesis. In this work, the physical modification technique was adapted to tailor MCM-48 particle size by varying stirring speed (i.e., $1000 \mathrm{rpm}, 3000 \mathrm{rpm}$ and $5000 \mathrm{rpm}$ ) and using different types of washing medium. Particle size distribution (PSD) studies showed mean particle size of $250 \pm 50 \mathrm{~nm}$. XRD analysis displayed several crystalline peaks indexed to cubic crystal mesophase. Transmission electron microscopy (TEM) and Brunauer-Emmett Teller (BET) analyses also showed a unique $3 D$ cubic interconnecting porous network with specific surface area of $1030 \mathrm{~m}^{2} \mathrm{~g}^{-1}$. It was revealed that the degree of agglomeration increased as relative polarity of alcohol increased, $\mathrm{CH}_{3} \mathrm{OH}>\mathrm{CH}_{3} \mathrm{CH}_{2} \mathrm{OH}$. Therefore, particle size and agglomeration state of MCM-48 can be tailored by varying the physical modification techniques while maintaining its intrinsic pore morphology.
\end{abstract}

Keywords: Sol-gel silica, agglomeration, MCM-48, nanosized, stirring speed 


\section{INTRODUCTION}

In the past two decades, ordered mesoporous silica (OMS) has inspired prominent research interest due to its unique attributes, facile synthesis and ease of functionalisation. Although OMS is considered a conventional filler, it is found to be widely explored in numerous fields due to its tunable pore and surface properties. ${ }^{1-3}$ OMS can be integrated into an organic polymer matrix to fabricate polymer nanocomposites or mixed matrix membranes (MMMs). OMS can be categorised into a class of mesoporous silica member including: Mobil Composition of Matter (MCM- $n$ ), $n=41,48$ and 50; Santa Barbara (SBA- $n$ ), $n=15,16$; and Korea Advanced Institute of Technology (KIT- $n$ ), $n=6 \cdot{ }^{4-6}$ OMS is driven by two primary factors: (1) self-assembly of surfactant micelles in lyotropic liquid crystals (LLCs); and (2) cationic exchange between negatively charged silicate species and positively charged head group of surfactants. ${ }^{7-11}$ M41S family can be differentiated according to the space group such as 2D-hexagonal $(\mathrm{p} 6 \mathrm{~mm})$ and gyroidal cubic Ia3d for MCM-41 and MCM-48, respectively. ${ }^{6,12,13}$ Highly crystalline cubic mesoporous materials possess a perfectly ordered and symmetric pore geometry of cubic gyroidal structure ( $G$-surface) ${ }^{14}$ The pore structure of hexagonal and gyroid cubic ordered porous arrays are represented in Figure 1.

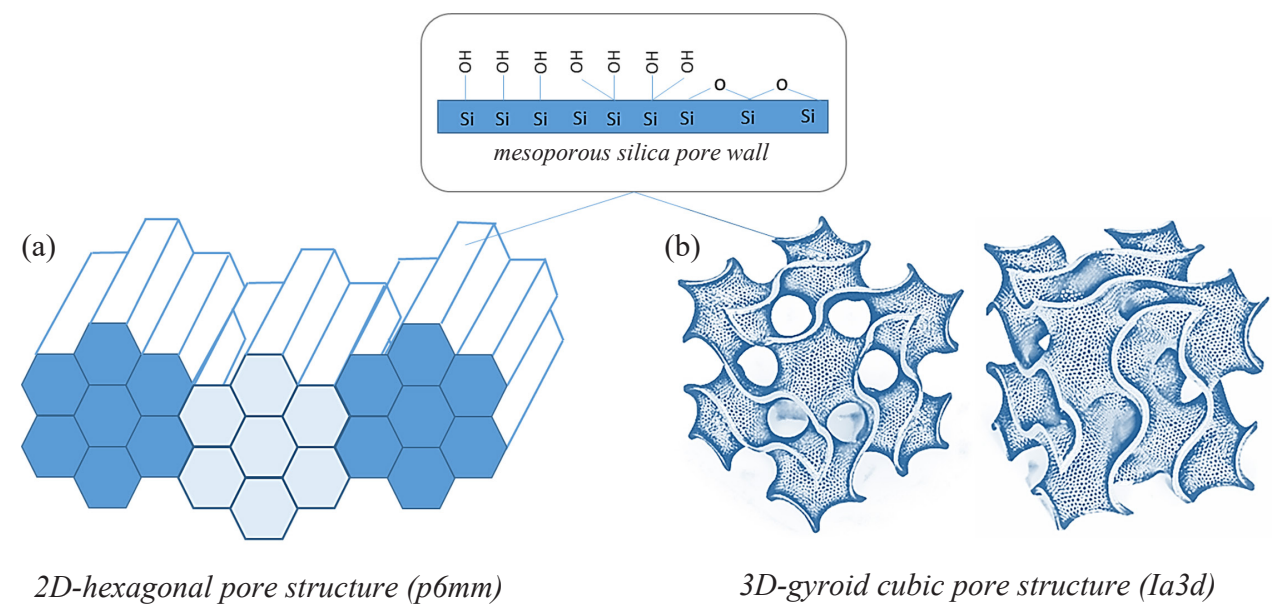

Figure 1: Structures of ordered mesoporous materials, M41S: (a) MCM-41 (2D hexagonal, space group $p 6 \mathrm{~mm}$ ), and (b) MCM-48 (cubic, space group Ia3d).

To date, both hydrothermal and room temperature synthetic condition can be applied for the synthesis of mesoporous silica. The primary difference between the hydrothermal and room temperature syntheses are reaction temperature, reactant composition, stirring condition, aging time, and the use of co-surfactant as structure 
directing agent. The formation of mesoporous silica is dependent on the nature of surfactants such as alkyl chain length and polarity. The surfactant(s) is crucial in determining surfactant packing parameter $g=V / a_{o} l$, where $V$ is the volume of the hydrophobic chain, $a_{o}$ is the effective area of the polar head group and $l$ is the alkyl chain length of surfactant. ${ }^{11}$ For instance, the packing parameter of gyroidal cubic mesophase surfactant ( $G$-surface) should be inherently controlled at surface curvature, $g=1 / 2<g<2 / 3$. Generally, silica growth is governed by the rate of silica hydrolysis and condensation. These parameters should be controlled to prevent the formation of secondary particles with larger particle size. ${ }^{15}$ Besides that, during silica growth, modification such as the addition of co-surfactant or $\mathrm{pH}$ adjustment can be performed to tailor the growing silicate species. However, other complications might arise such as the presence of other undesired mesophase or structure collapse. Therefore, tailoring of MCM-48 via physical modification provides a better option to reduce the particle size. For instance, hexagonal mesoporous silica with reduced particle size has been successfully achieved by tailoring the stirring speed up to $500 \mathrm{rpm}$. In comparison with MCM-48, stirring speed up to $850 \mathrm{rpm}$ has been applied to single surfactant system at room temperature. It was reported that particle size of MCM-48 synthesised at 450-850 rpm produced particle size distribution between $200 \mathrm{~nm}$ and $1000 \mathrm{~nm} .{ }^{16-20}$ It is hypothesised that longer duration of mechanical stirring can induce the formation of larger secondary silica particle due to interparticle collision. To date, Kim and his co-workers successfully synthesised nanosized MCM-48 spheres using double surfactant system, i.e., cationic surfactant and non-ionic block-copolymer. ${ }^{21}$ The usage of optimum stirring speed and stirring time could minimise the agglomeration of particles. By adapting to this method, synthesis of nanosized MCM-48 can be achieved using minimal amount of silica precursor at a controlled rate of silica addition, under single cationic surfactant system. In this work, stirring speed of disperser was manipulated to tailor the particle size of MCM-48 via single surfactant system. The study was extended to evaluate the effect of washing medium towards the size of MCM- 48 particle agglomerates.

\section{EXPERIMENTAL}

\subsection{Materials Selection}

Cetyltrimethylammoniumbromide (CTAB), 35\% aq. $\mathrm{NH}_{4} \mathrm{OH}$, ethanol, tetraethylorthosilicate (TEOS) were purchased from Merck, KGaA, Germany. Polyethylene glycol tert-octyl phenyl ether (Triton ${ }^{\mathrm{TM}} \mathrm{X}-100$ ) was obtained from Sigma-Aldrich. All chemicals were used without further purification. 


\subsection{Synthesis of MCM-48}

\subsubsection{Effect of surfactant type on formation of MCM-48}

Synthetic solutions with single and double surfactant system were prepared to study favourable condition to synthesise cubic mesostructured siliceous MCM-48. The reactant composition is shown in Table 1.

Table 1: Molar composition at room temperature for single and double surfactant system.

\begin{tabular}{clll}
\hline Sample & Synthesis condition & Surfactant composition & Molar composition mixture \\
\hline S1 & Single surfactant & $0.41 \mathrm{CTAB}$ & $0.41 \mathrm{CTAB} / 11 \mathrm{aq} . \mathrm{NH}_{3} / 1.0 \mathrm{TEOS} / 53$ \\
& & & EtOH/344 $\mathrm{H}_{2} \mathrm{O}$ \\
& & $1.0 \mathrm{TEOS} / 0.139 \mathrm{CTAB} / 0.028$ \\
S2 & Double surfactant & $0.139 \mathrm{CTAB}:$ & $\begin{array}{l}\text { Triton } \\
\end{array}$ \\
& & $0.028 \mathrm{Tx}-100$ & $\mathrm{X}-100 / 0.468 \mathrm{NaOH} / 54.7 \mathrm{H}_{2} \mathrm{O}$ \\
\hline
\end{tabular}

\subsubsection{Effect of synthesis condition (room temperature vs. hydrothermal) on MCM-48 formation}

The experimental study on the effect of synthesis condition towards the formation of MCM-48 silica was executed at room temperature and hydrothermal condition. The system was designed to determine the optimum reaction temperature for gyroidal cubic MCM-48 formation. Referring to Table 2, single surfactant system was prepared by using cetyltrimethylammonium bromide (CTAB) as templating medium, ethanol (co-solvent), ammonium hydroxide (morphology catalyst), sodium hydroxide (catalyst) and deionised water (solvent). Particle shape and size distribution for the as-synthesised MCM-48 were studied via scanning electron microscopy (SEM) and particle size analyser (PSA).

Table 2: Molar composition of mixture under single surfactant system at different synthesis conditions.

\begin{tabular}{cll}
\hline System & Synthesis condition & Molar composition of mixture $(\mathrm{M})$ \\
\hline S3 & Room temperature & $1 \mathrm{M} \mathrm{TEOS} / 12.5 \mathrm{M}$ aq. $\mathrm{NH}_{3} / 54 \mathrm{M} \mathrm{EtOH} / 0.4 \mathrm{M}$ \\
& & $\mathrm{CTAB} / 174 \mathrm{M} \mathrm{H}_{2} \mathrm{O}$ \\
& & $1 \mathrm{M} \mathrm{TEOS} / 0.25 \mathrm{M} \mathrm{Na} \mathrm{N}_{2} \mathrm{O} / 0.65 \mathrm{M}$ \\
S4 & Hydrothermal & $\mathrm{CTAB} / 0.62 \mathrm{M} \mathrm{H}_{2} \mathrm{O}$ \\
\hline
\end{tabular}




\subsubsection{Effect of synthetic solution composition on MCM-48 formation}

Under single surfactant system, four different samples were prepared by varying the reactant ratios of surfactant/silica, water/silica, water/ammonia (aq.) and ethanol/surfactant as displayed in Table 3.

Table 3: Molar composition of varied reactants ratio in single surfactant system.

\begin{tabular}{ccccc}
\hline Sample no. & CTAB/TEOS & $\mathrm{H}_{2} \mathrm{O} /$ TEOS & $\mathrm{H}_{2} \mathrm{O} / \mathrm{NH}_{3}$ aq. & EtOH/CTAB \\
\hline S5 & 0.4 & 174 & 13.92 & 135 \\
$\mathrm{~S} 6$ & 0.38 & 935 & 14.60 & 101 \\
$\mathrm{~S} 7$ & 0.41 & 344 & 31.27 & 129.3 \\
$\mathrm{~S} 8$ & 0.64 & 1668 & 33.36 & 135 \\
\hline
\end{tabular}

\subsubsection{Effect of stirring speed and type of washing solvent}

Based on predetermined reactant composition, S8 experimental condition was adopted to tailor MCM-48 by varying the stirring speed (Table 4). The experimental setup and process flow were displayed in Figure 2. First, the synthetic solution was prepared by dissolving $\mathrm{CTAB}$ in deionised water at $250 \mathrm{rpm}$ before adding ethanol and aqueous ammonium hydroxide. After $15 \mathrm{~min}$, the solution was transferred to disperser and TEOS was added dropwisely at $0.15 \mathrm{~cm}^{3} \mathrm{~s}^{-1}$. Then, the homogeneous mixture was aged for $24 \mathrm{~h}$. The solid precipitates were recovered and the CTAB templates were removed at $550^{\circ} \mathrm{C}$ for $6 \mathrm{~h}$. Post treatment of MCM- 48 was executed using different washing medium to reduce the agglomeration of particles. It is hypothesised that strong polarity washing medium such as water, normally causes the particles to stick and clump together after complete drying.

Table 4: Stirring speed of disperser in single surfactant system, aged for $24 \mathrm{~h}$ with ethanol washing medium.

\begin{tabular}{cc}
\hline Sample trial & Stirring speed $\left(\mathrm{rev} \mathrm{min}^{-1}\right)$ \\
\hline S9 & 1000 \\
S10 & 3000 \\
S11 & 5000 \\
\hline
\end{tabular}




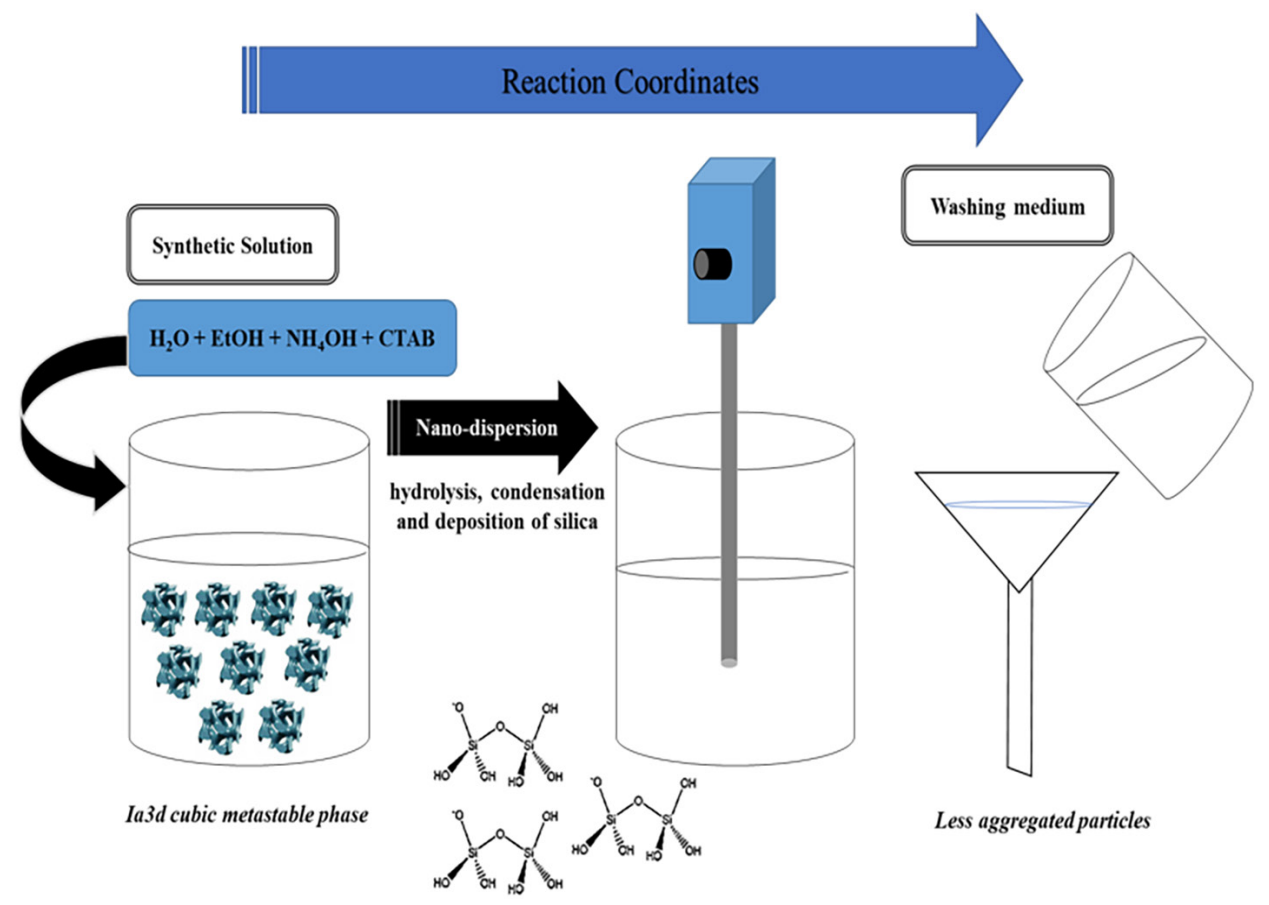

*Synthetic solution consisting lyotropic liquid crystals (LLCs): driven by surfactants self-assembly and transformation to isotropic cubic mesophase Ia3d, $1 / 2<\mathrm{g}<2 / 3$ (G-surface)

Figure 2: Reaction coordinates of MCM-48 tailored at varied stirring speed and washing medium.

\subsection{Effect of Rotational Motion and Vortex Pattern for Formation of MCM-48 Nanoparticles}

T-25 Ultra Turrax Disperser with 1000-5000 rpm is employed. This disperser is expected to homogenise and ensure better dispersion of silicates during solgel silica process. By controlling the stirring speed and stirring time, stable nanoparticles will be produced. Additionally, the unique sawtooth design allows large particles (agglomerates) to dissociate at high circumferential speed as shown in Figure 3. However, the resulting shear force and inward rotating mechanism might disrupt the micelle-templating solution. Therefore, stirring time must be kept at a minimum to allow formation of micelle-silicate complex and silica deposition by ion-exchange mechanism (i.e., $\mathrm{Br}^{-}$is substituted by $\mathrm{Si}^{-} \mathrm{O}^{-}$). 


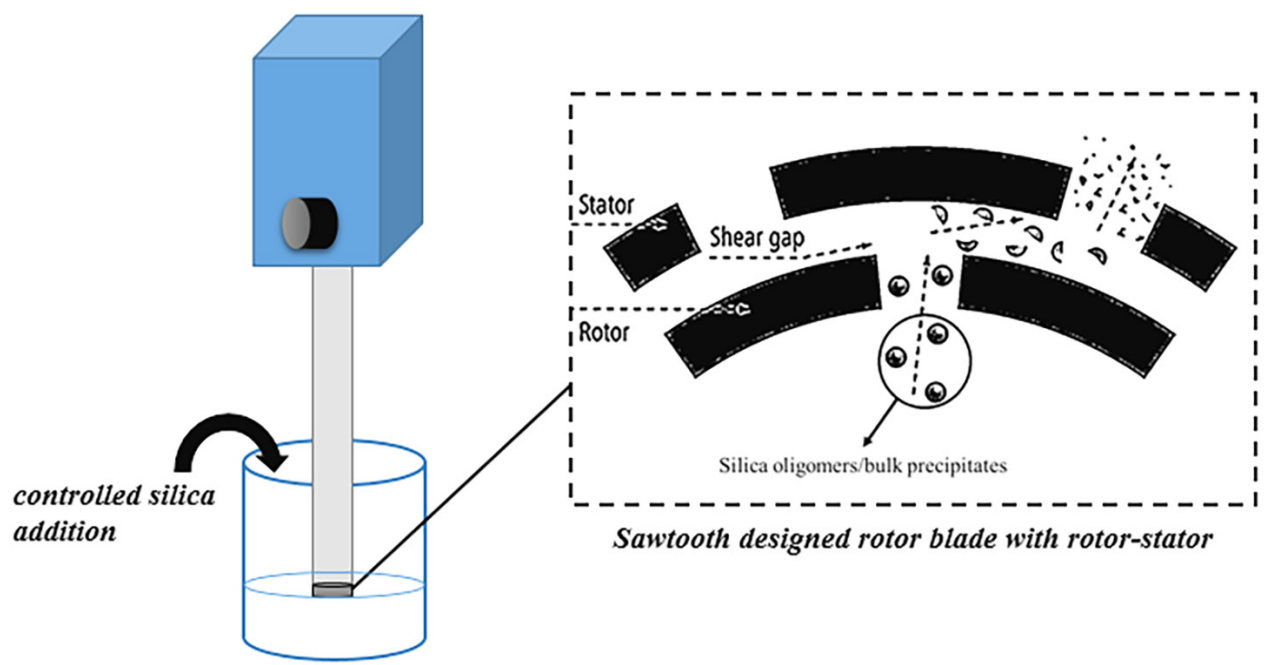

Figure 3: Illustration on underlying rotating mechanism of disperser used in sol-gel silica process.

\subsection{Characterisation of MCM-48}

X-ray diffraction data of the MCM-48 spheres were obtained using $X^{\prime}$ Pert $^{3}$ Powder, PANalytical, with $\mathrm{Cu} \mathrm{K} \alpha$ radiation wavelength of $\alpha=0.154 \mathrm{~nm}$ in the 2-theta range of $2^{\circ}-8^{\circ}$ with a scan speed of $1^{\circ} \mathrm{min}^{-1}$. The morphology of MCM-48 was examined from SEM and field emission SEM (FESEM) images using Bruker Nano EDSHitachi TM3000 Tabletop Microscope (GmbH, Germany) and VPFESEM, Zeiss Supra55 VP. For the imaging analysis, the sample was deposited on a sample holder with an adhesive carbon foil and sputtered with platinum. Mean particle size and particle size distribution analyses were conducted using PSA, Mastersizer 2000. BET surface analysis was performed using Micromeritics TriStar II. The samples were degassed at $250^{\circ} \mathrm{C}, 1.382$ atm for $6 \mathrm{~h}$ prior to measurements. Nitrogen adsorption and desorption isotherms were measured at liquid nitrogen temperature of $-195.8^{\circ} \mathrm{C}$. TEM (Tecnai TF20 X-Twin) FEI was employed to analyse the pore geometry of MCM-48 at different incidence planes. The pore geometry and crystal structure of tailored MCM-48 were investigated by tilting the crystals along [111] and $[110]$ zone axes. 


\section{RESULTS AND DISCUSSIONS}

\subsection{Effect of Reaction Parameters: Surfactant Type and Synthesis Condition}

\subsubsection{The effect of surfactant type towards formation of gyroidal cubic MCM-48}

The XRD of MCM-48 synthesised via single and double surfactant systems were displayed in Figure 4. The result for single surfactant system (S1) is in agreement with Bragg's peaks definition for bicontinuous pore network ascribed to gyroidal cubic mesophase. In contrast to S1, S2 which represents double surfactant system showed lack of observable peak at (220), as shown in Figure 4(b). It is hypothesised that the major peak (220) did not appear due to co-existence of other mesophases like hexagonal or lamellar or both. S1 showed well-resolved major Bragg's peaks at $2 \theta=2.65^{\circ}, 3.07^{\circ}$, and minor peaks at $4.33^{\circ}, 4.52^{\circ}, 4.94^{\circ}$ and $5.07^{\circ}$, as shown in Figure 4(a). This clearly indicated that S1 has a highly ordered cubic mesoporous structure, defined at (211), (220), (400), (421), (332) and (431) and consistent with Ia3 $d$ cubic space group. ${ }^{22-26}$ It showed that single surfactant system was favorable because higher degree of crystallinity for cubic mesoporous silica was obtained.
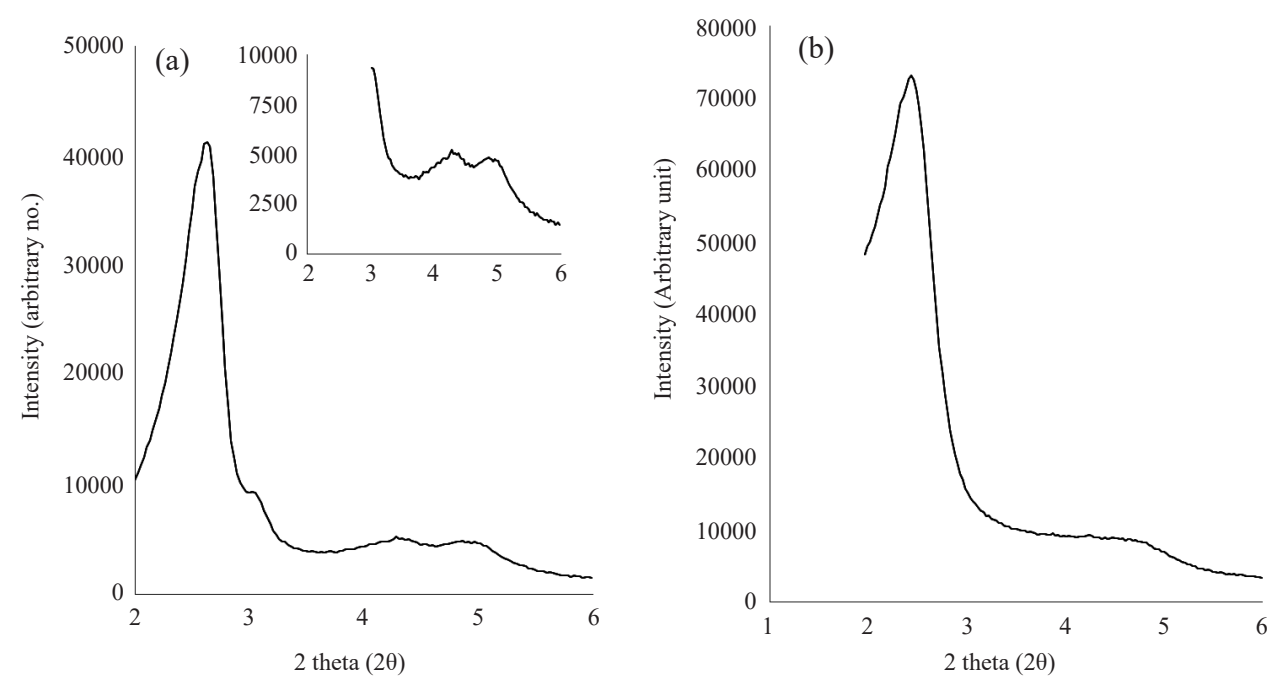

Figure 4: XRD diffraction pattern for MCM-48 using (a) single surfactant system S1, and (b) double surfactant system S2. 


\subsubsection{Physical morphology of MCM-48 via single and double surfactant system}

Electron microscopy image of MCM-48 shows the distribution of spherical MCM48 particles formed at room temperature and hydrothermal condition (Figure 5). It was found that double surfactant system shows enhanced monodispersity and particle size compared to single surfactant. It can be deduced that the growth of particles in single surfactant system is due to deposition of primary particles to the stable particle and formation of agglomerates. It may also be due to interparticle contact by collision and aging duration. Longer stirring time and interparticle contact lead to permanent aggregation due to hydrophilic surface of silica.
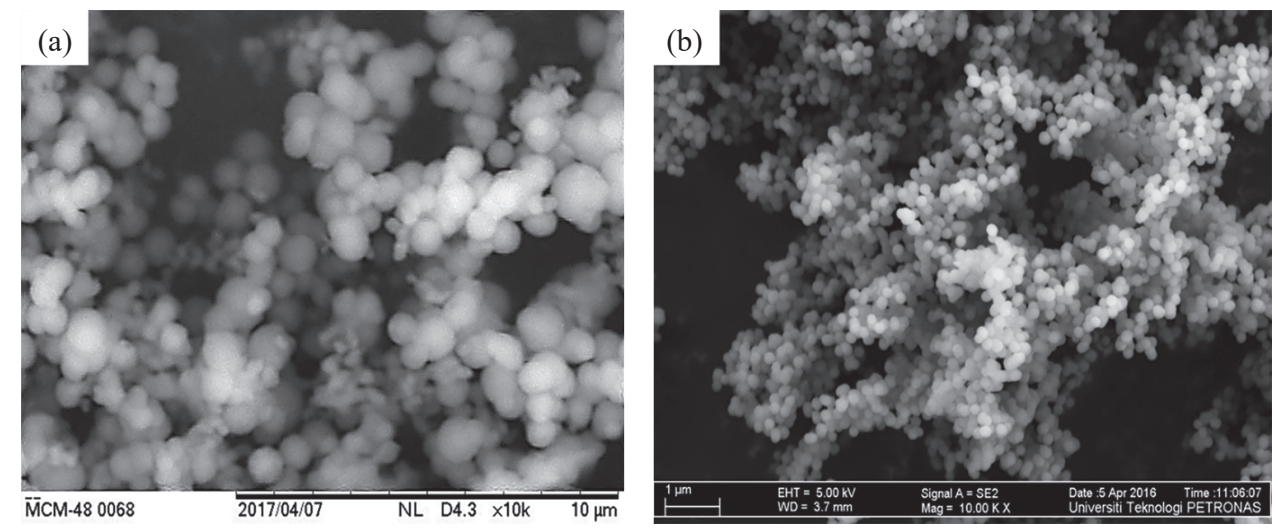

Figure 5: Electron microscopy images of MCM-48 at 10K magnification using (a) single surfactant, S1, and (b) double surfactant, S2.

\subsubsection{The effect of surfactant type on the structure of cubic MCM-48}

TEM images confirmed the structure of cubic mesopores for S1. Referring to Figure 6, S1 displayed the intrinsic cubic $I \mathrm{a} 3 \mathrm{~d}$ mesopores from incidence plane [111]. As compared to S1, S2 barely displayed any pore formation and no structural order of cubic Ia $3 d$ was observed as well. In spite of that, a lettuce-like or layered form of pore structure possessed by the lamellar material was observed. This might be due to several factors such as the use of co-surfactant, $\mathrm{pH}$ changes, reaction temperature and aging time. ${ }^{27-29}$ Even though it results in monodispersed silica sphere and uniform particle size, double surfactant system is not favourable for MCM-48 formation. This is mainly due to extremely sensitive templating solution, wherein a slight change in surfactant volume or chain length will cause the formation of undesired mesophase. Hence, single surfactant system is easier to control in order to further optimise the synthetic condition. 

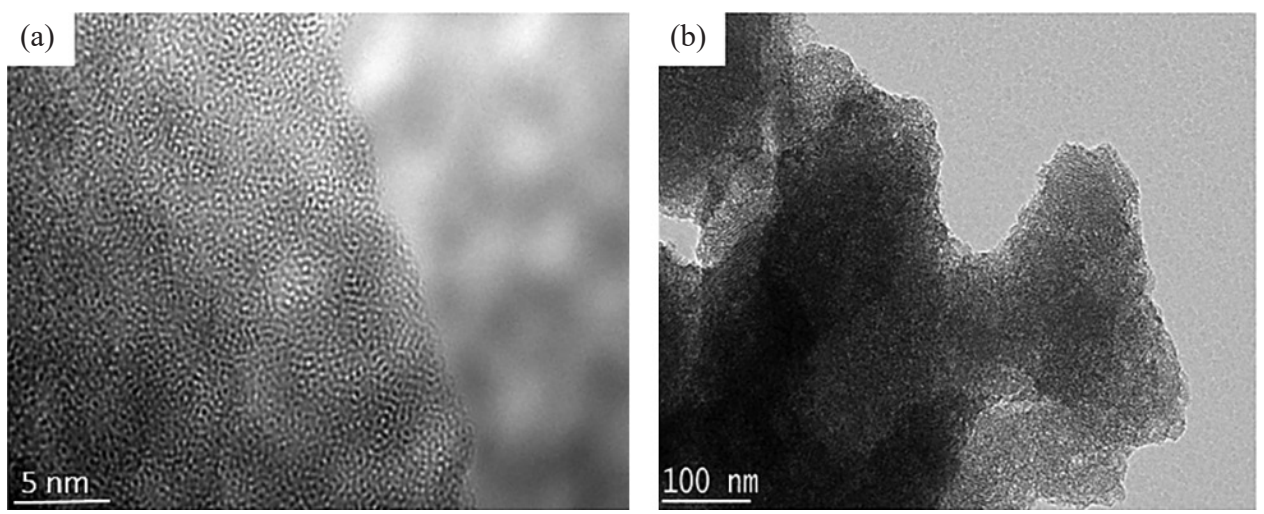

Figure 6: HRTEM micrographs of MCM-48 in (a) single surfactant S1, and (b) double surfactant S2.

\subsubsection{Effect of synthesis condition (hydrothermal vs. room temperature) on MCM-48 via $\mathrm{N}_{2}$ isotherm studies}

Referring to Figure 7, S3 and S4 showed a typical Type IV nitrogen adsorptiondesorption isotherm. However, capillary condensation accompanied with adsorption hysteresis was observed at $\mathrm{p} / \mathrm{p}^{\circ} 0.6-0.9$ for S3. This occurs when the pore diameter of $43.84 \dot{\mathrm{A}}$ exceeds a certain critical width as revealed by $\mathrm{BJH}$ desorption at $196.15^{\circ} \mathrm{C}$. In principle, hysteresis occurs when the pores are wider than $40 \dot{\mathrm{A}}$. In contrast, S4 revealed a reversible isotherm of Type IV., 40,31 $\mathrm{S} 4$ has smaller pore size $(28.36 \dot{\mathrm{A}})$ than S3. The pore size of S4 is too large for mesoporous silica, which occurs as a result of change in surfactant volume. The double surfactant system which employed cationic and non-ionic surfactant, experienced a drastic change in hydrophobic surfactant chain length $(l)$, leading to pore-expansion of cubic silica mesophase. It is hypothesised that this phenomenon might be induced by addition of hydrochloric acid during micelles-silicates complex which alters the system equilibrium. Overall pore properties such as BET surface area, pore volume, and pore size are presented in Table 5. S4 has larger surface area than $\mathrm{S} 3$, along with a slight increase in pore volume and smaller pore size or diameter. Hence, this concludes that mesoporous system of gyroidal cubic Ia $3 d$ silica is favoured at room temperature. 

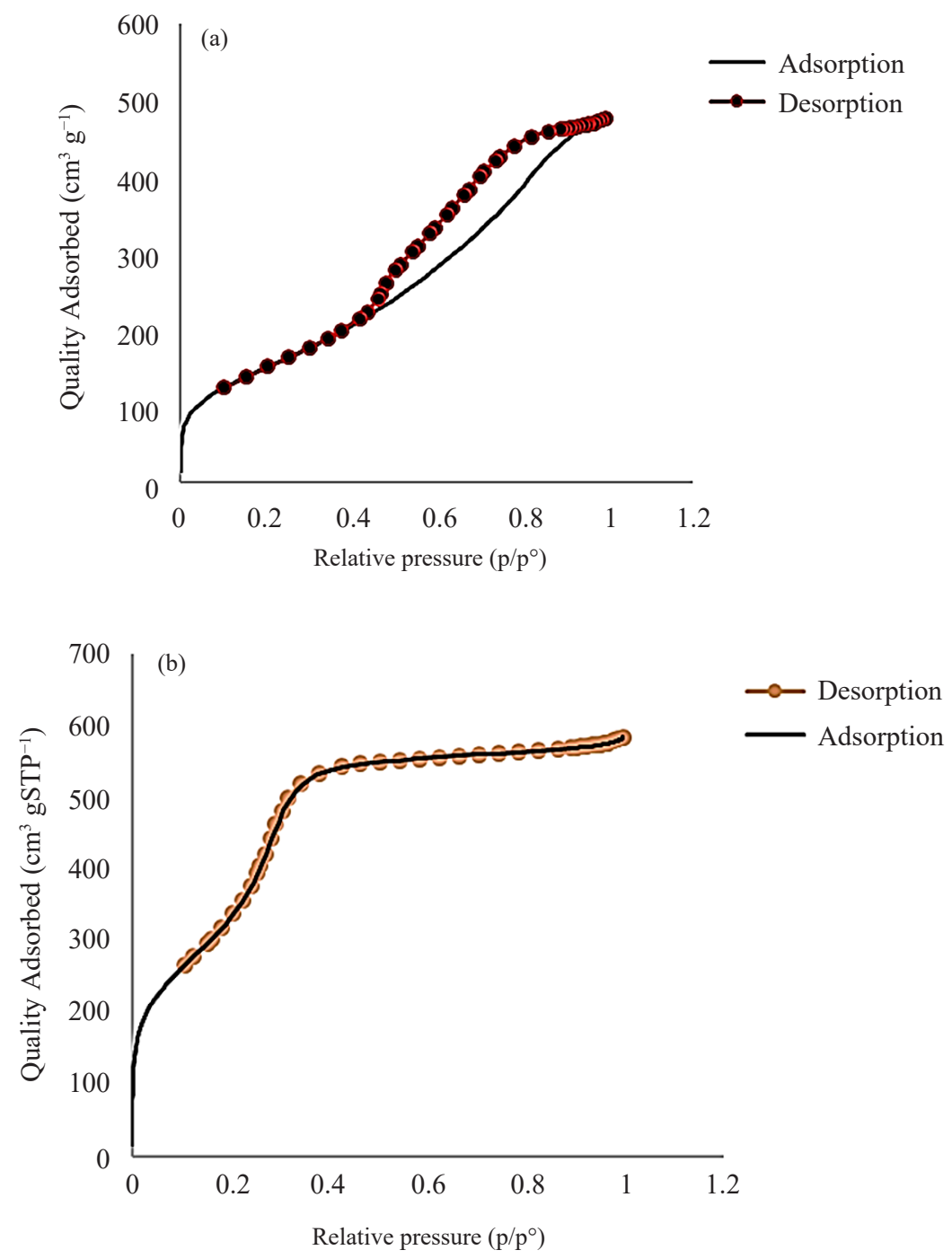

Figure 7: Nitrogen adsorption-desorption isotherms for MCM-48 synthesised at (a) hydrothermal S3, and (b) room temperature S4.

Table 5: BET surface area, pore volume and pore size of MCM-48.

\begin{tabular}{lccc}
\hline Sample trial & $\begin{array}{c}\text { BET surface area } \\
\left(\mathrm{m}^{2} \mathrm{~g}^{-1}\right)\end{array}$ & $\begin{array}{c}\text { BJH desorption } \\
\text { pore volume }\left(\mathrm{cm}^{3} \mathrm{~g}^{-1}\right)\end{array}$ & $\begin{array}{c}\text { BJH desorption } \\
\text { pore size }(\dot{\mathrm{A}})\end{array}$ \\
\hline Hydrothermal, S3 & 550.79 & 0.766 & 43.84 \\
Room temperature, S4 & 1108.24 & 0.797 & 28.36 \\
\hline
\end{tabular}




\subsubsection{Effect of synthesis condition (hydrothermal vs. room temperature) on particle size distribution of MCM-48}

The particle size distribution (PSD) for MCM-48 synthesised at hydrothermal, S3 and room temperature, $\mathrm{S} 4$ revealed bimodal distribution. The particles size range for S3 and S4 are 0.7-1.0 $\mu \mathrm{m}$ and 0.9-2.0 $\mu \mathrm{m}$, respectively. Although S3 exhibits smaller particle size, BET isotherm in Figure 8(a) showed undesirable pattern indicating weak adsorbent-adsorbate interaction.
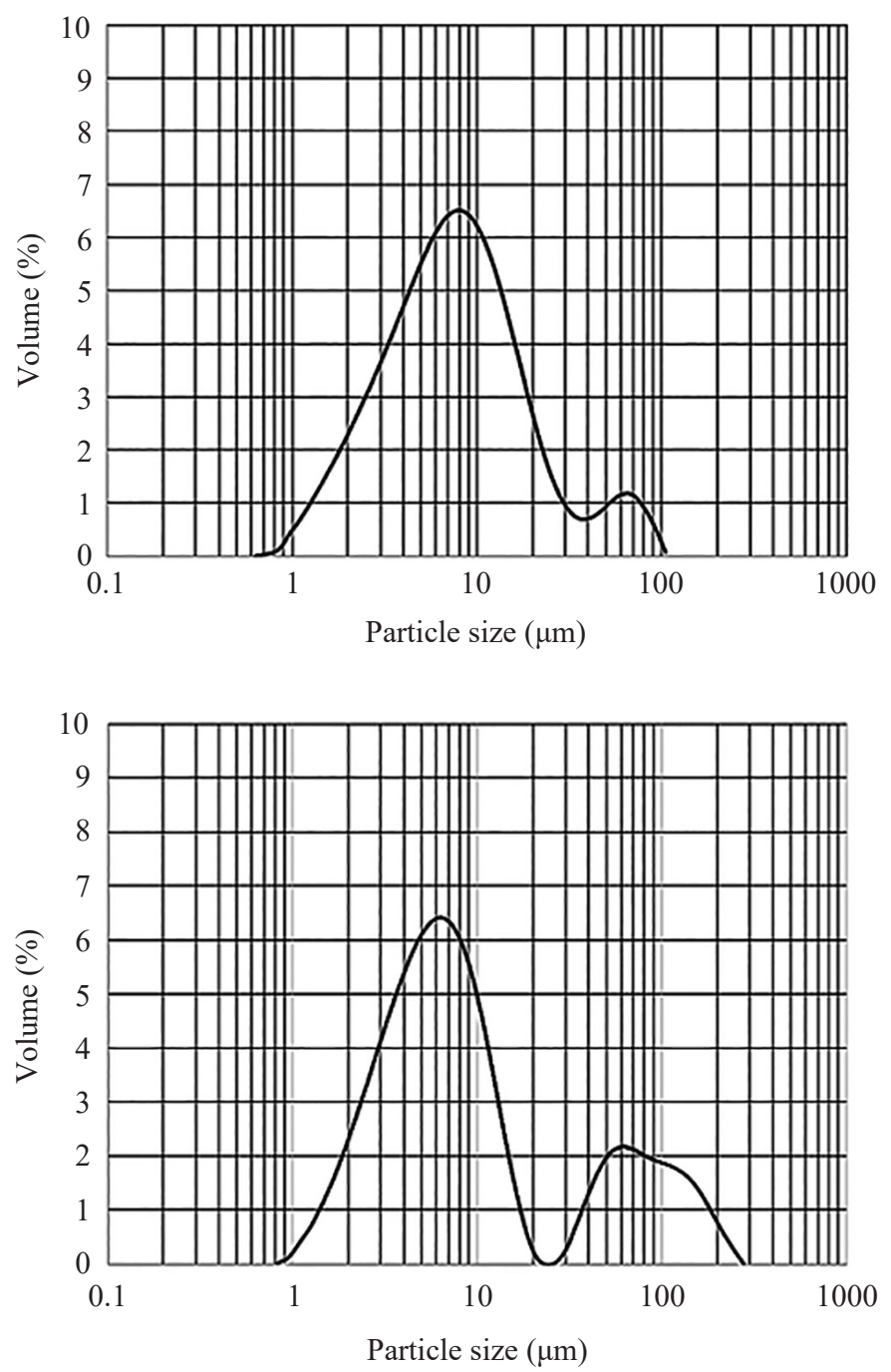

Figure 8: Particle size distribution by volume (\%) of MCM-48 synthesised via (a) hydrothermal S3, and (b) room temperature S4. 


\subsubsection{Effect of synthesis condition (hydrothermal vs. room temperature) on particle morphology of MCM-48}

SEM images of MCM-48 prepared via room temperature (RT) synthesis showed the formation of MCM-48 with uniform spherical morphology whilst hydrothermally (HT) synthesised MCM-48 showed irregular particles(Figure 9). It was hypothesised that spherical morphology is due to the presence of ammonium hydroxide which not only acts as a basic catalyst but also as a morphological catalyst. To date, spherical shaped MCM-48 is easier to disperse in a wet medium like polymer dope solution, thus making it feasible as an inorganic filler for fabrication of filled polymer or composite membrane film. Furthermore, by reducing the possibility of surface contact within the particles, surface cohesiveness could be diminished due to polar and van der Waals attraction of hydrophilic silica.

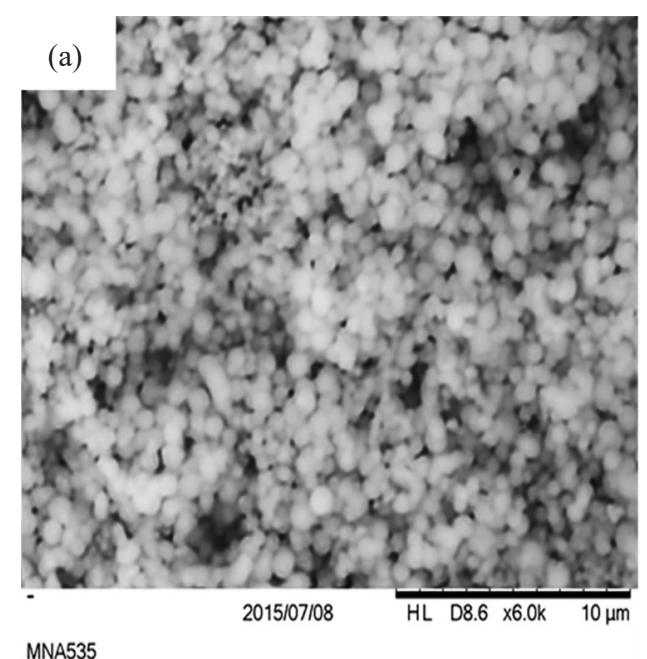

MNA535

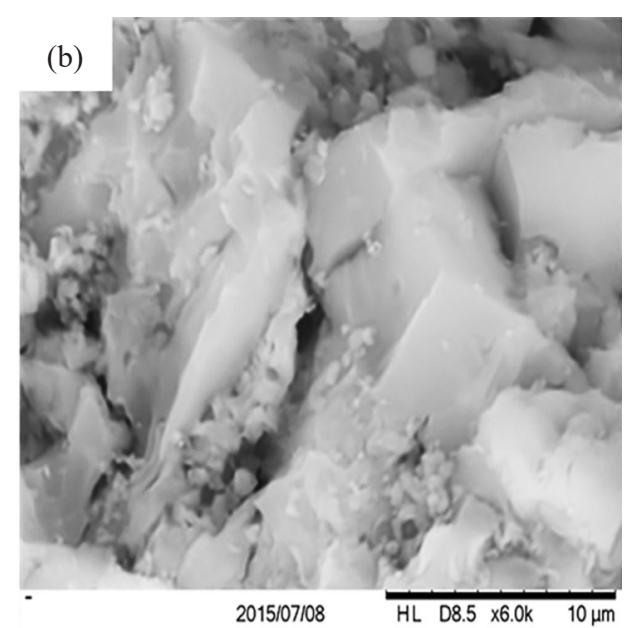

MNA535

Figure 9: SEM images of MCM-48 (6K magnification) at (a) hydrothermal S3, and (b) room temperature $\mathrm{S} 4$.

\subsubsection{Effect of synthetic solution composition on MCM-48 particle morphology}

The physical morphology of MCM-48 was studied by using SEM and FESEM to ascertain the particle shape, size and aggregation states of each sample. Based on the water to silica composition ratio and cetyltrimethylammonium bromide to tetraethylorthosilicate ratio, different morphological patterns of MCM-48 were observed. In Figure 10(a-d), MCM-48 particle morphologies were seen as: (a) smaller and monodispersed spherical particles; (b) a combination of large and 
small particles with fused-like shapes and aggregated particles; (c) monodispersed particles with the presence of smaller unresolved and irregular shapes; and (d) spherical particles with ellipsoidal shaped-like structures in uniform size ranges. Based on microscopic images, it can be concluded that $\mathrm{S} 8$ yields the most desired MCM-48 morphology, indicating that reactant molar ratio played a crucial role in silica growth.
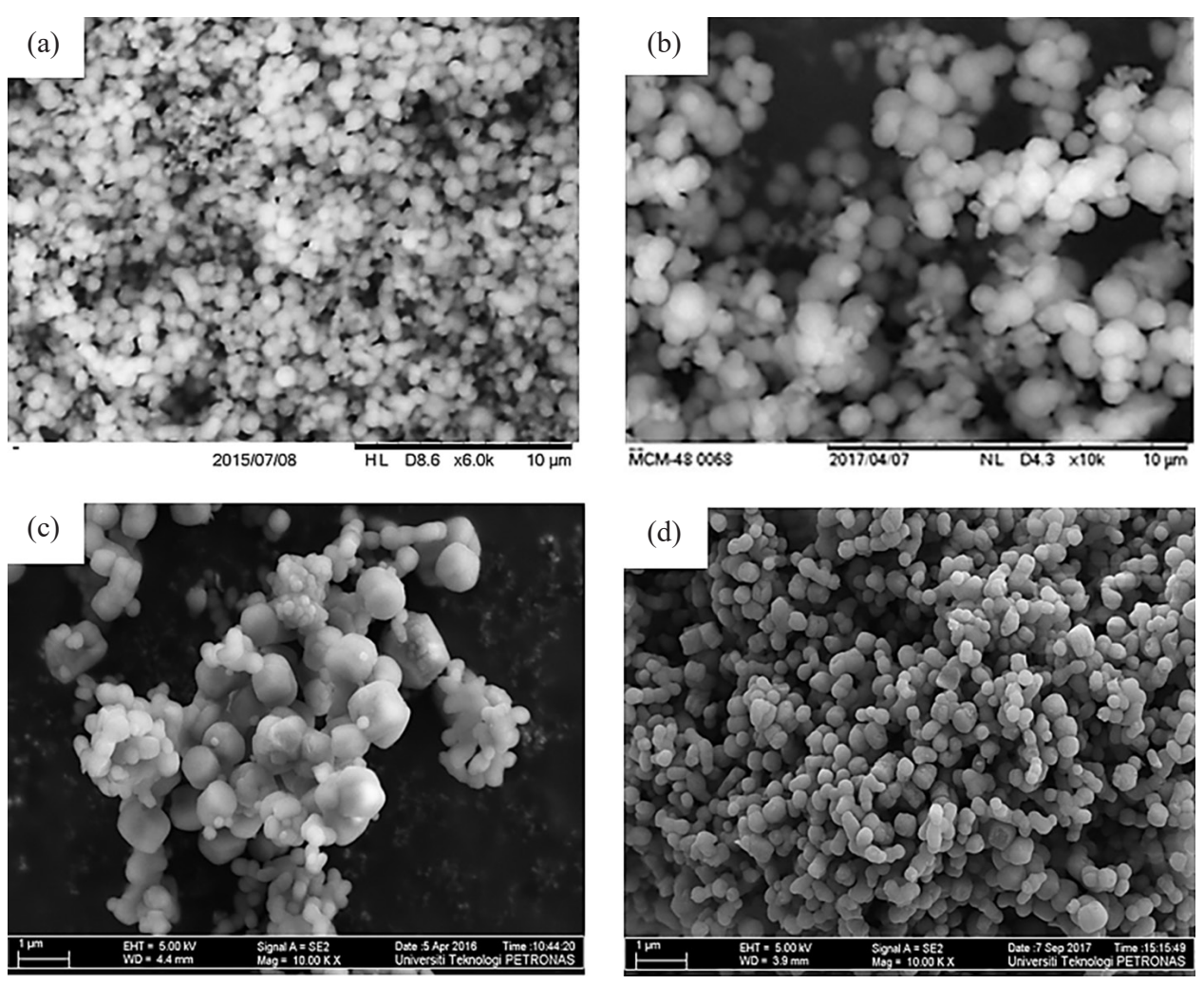

Figure 10: Electron microscopy images of (a) S5, (b) S6, (c) S7, and (d) S8 for MCM-48 at different reactant molar composition ratio synthesised in single surfactant system at room temperature.

\subsection{Tailoring MCM-48 via Optimised Sol-gel Silica Process}

\subsubsection{Effect of stirring speed on physical morphologies of MCM-48}

Figure 11 displays the morphology of tailored MCM-48. The as-synthesised and calcined MCM-48 tailored at $5000 \mathrm{rpm}$ showed uneven silica growth on stable particles. In contrast, MCM-48 synthesised at $1000 \mathrm{rpm}$ and $3000 \mathrm{rpm}$ yield similar 
particle morphology and aggregation states. PSD analysis presented in Figure 12 shows that $3000 \mathrm{rpm}$ is the optimum stirring speed to produce well-defined morphology of silica particles. Beyond that, it will cause undesired irregular silica particles formation since extreme agitation could disrupt the arrangement of micelle aggregates and facilitate agglomeration of siliceous MCM-48.
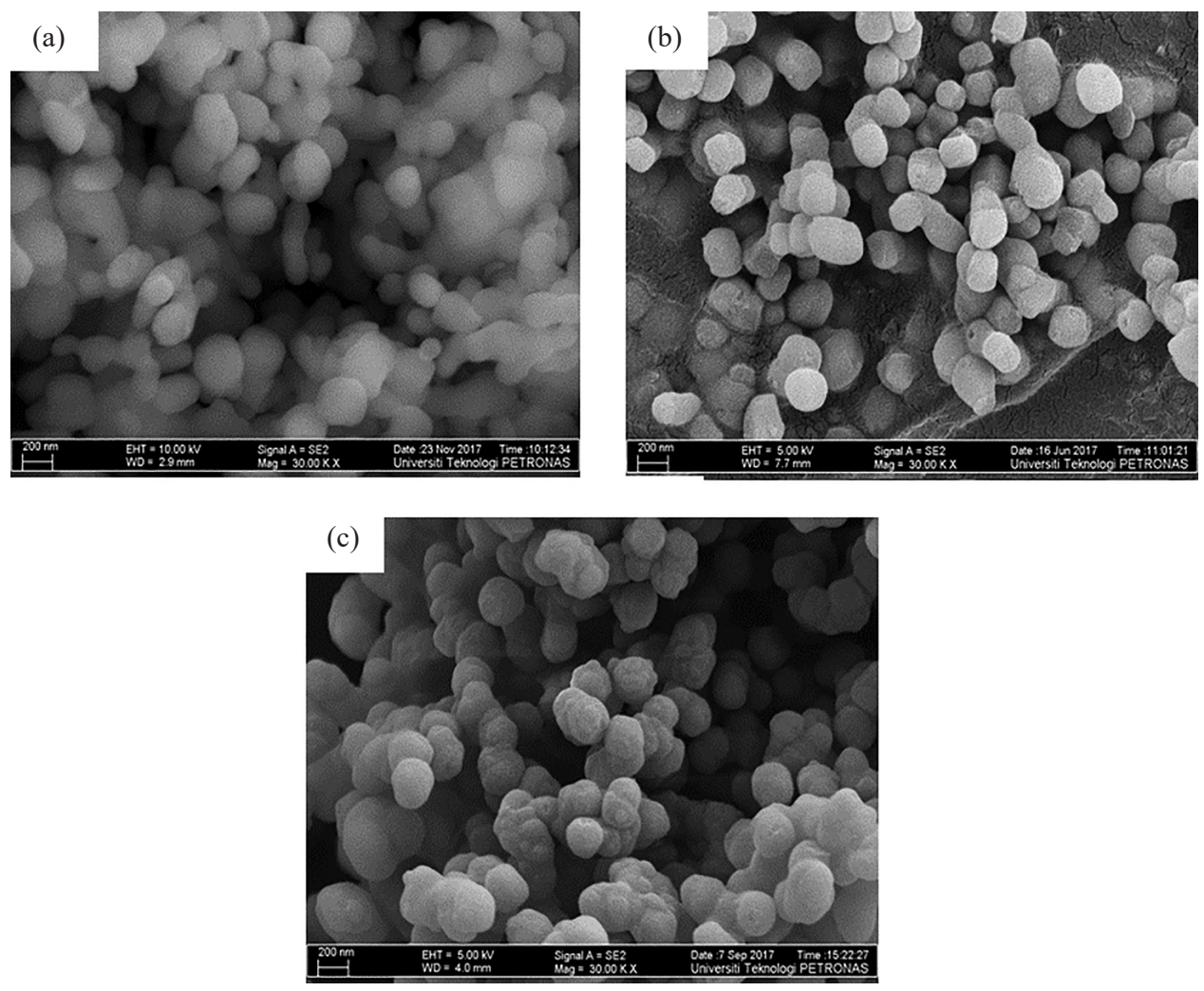

Figure 11: FESEM images of calcined MCM-48 viewed at 30K magnification for (a) S9: $1000 \mathrm{rpm}$, (b) S10: $3000 \mathrm{rpm}$, and (c) S11: $5000 \mathrm{rpm}$ washed with ethanol as washing medium.

MCM-48 silica tailored at $1000 \mathrm{rpm}$ (S9) and $3000 \mathrm{rpm}$ (S10) showed a narrow particle size distribution as compared to $5000 \mathrm{rpm}$ (S11). At $5000 \mathrm{rpm}, \mathrm{MCM}-48$ shows a broad range of particle size between $150 \mathrm{~nm}$ and $1 \mu \mathrm{m}$. Whilst for S9 and S10, the smallest particle sizes were determined at approximately $200 \mathrm{~nm}$ and $150 \mathrm{~nm}$, respectively. It was concluded that rigorous stirring speed possibly result in significant impact to silica growth and agglomeration of particles whereas the reduction in particle size was observed as stirring speed increased from $1000 \mathrm{rpm}$ to $3000 \mathrm{rpm}$. 

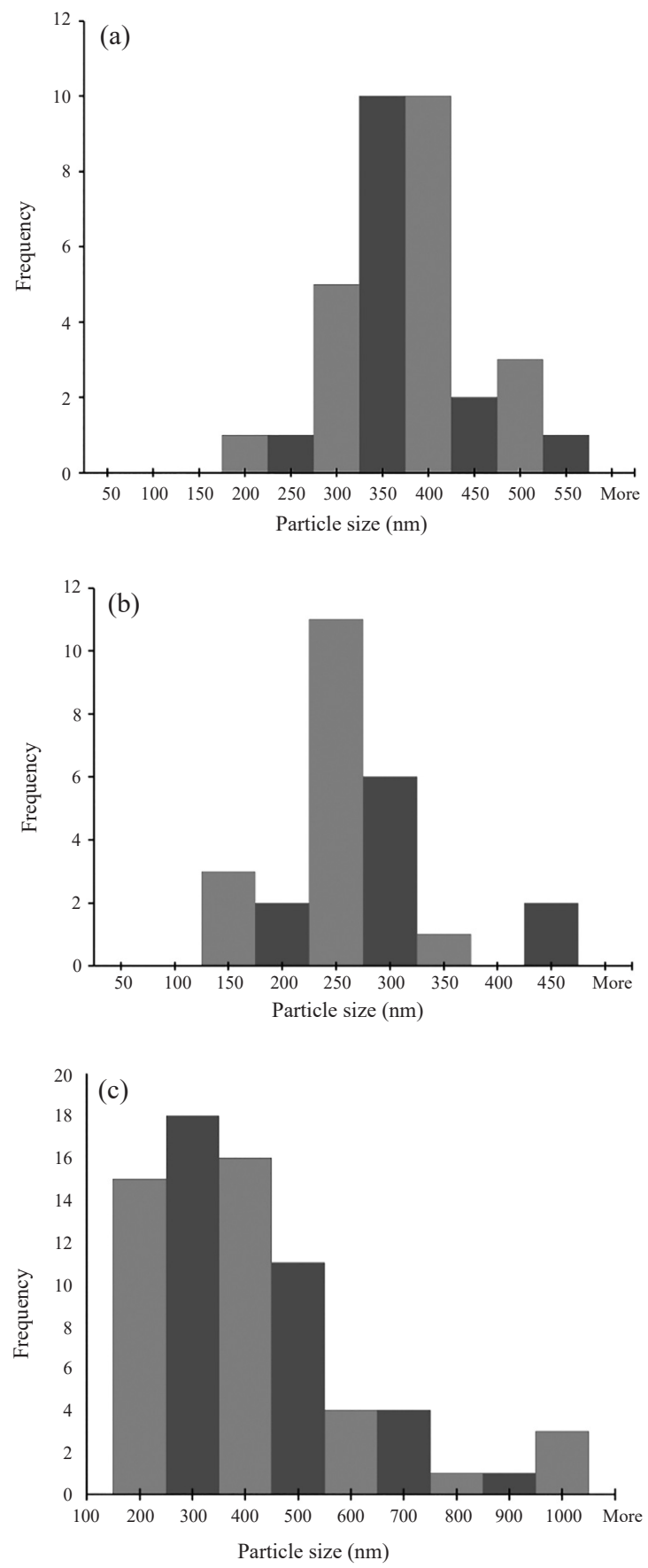

Figure 12: PSD of MCM-48 stirred at (a) $1000 \mathrm{rpm}, \mathrm{S} 9$, (b) $3000 \mathrm{rpm}, \mathrm{S} 10$, and (c) 5000 rpm, S11 washed with ethanol. 


\subsubsection{Effect of stirring speed to gyroidal cubic mesoporous structure}

Figure 13 shows the XRD diffraction pattern of the as-synthesised MCM-48 stirred at $3000 \mathrm{rpm}$. Two major significant peaks at $2 \theta=3.05$ and 3.42 were revealed at (211) and (220). The distortion and unresolved peak of (220) might be due to the presence of distorted portion of pore arrangement in MCM- 48 because of rigorous stirring procedure applied.

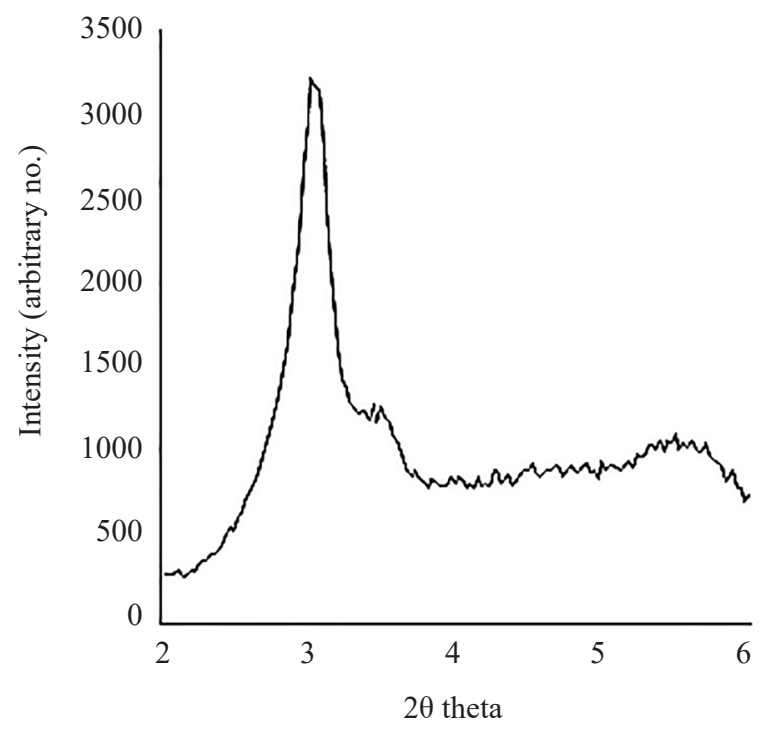

Figure 13: XRD diffraction of as-synthesised MCM-48 stirred at $3000 \mathrm{rpm}$ and washed with ethanol, S10.

\subsubsection{Pore mesostructure analyses of MCM-48 tailored at optimum stirring speed}

The HRTEM images for selected particles along the [111] and [110] plane axes are presented in Figure 14(a) which matched well with the reported MCM-48 images..$^{32,33}$ The [111] plane exhibited hexagonal-like porous structure which ascribed to the 211-type cubic structure. The plane [110] plane showed tetragonal crystal surface due to intersection of bicontinuous porous arrays. ${ }^{7}$ Both hexagonal and tetragonal structure represent a body-centred cubic (BCC) structure. As-synthesised MCM48 at $3000 \mathrm{rpm}$ showed a rhombohedral crystal surface morphology as seen in Figure 14(c). The SAED pattern in Figure 14(d) indicating a BCC mesostructure was formed. It is concluded that stirring speed variation within 1000 to $3000 \mathrm{rpm}$ is able to produce orderly arranged gyroidal cubic mesoporous silica arrays (Ia3d). 

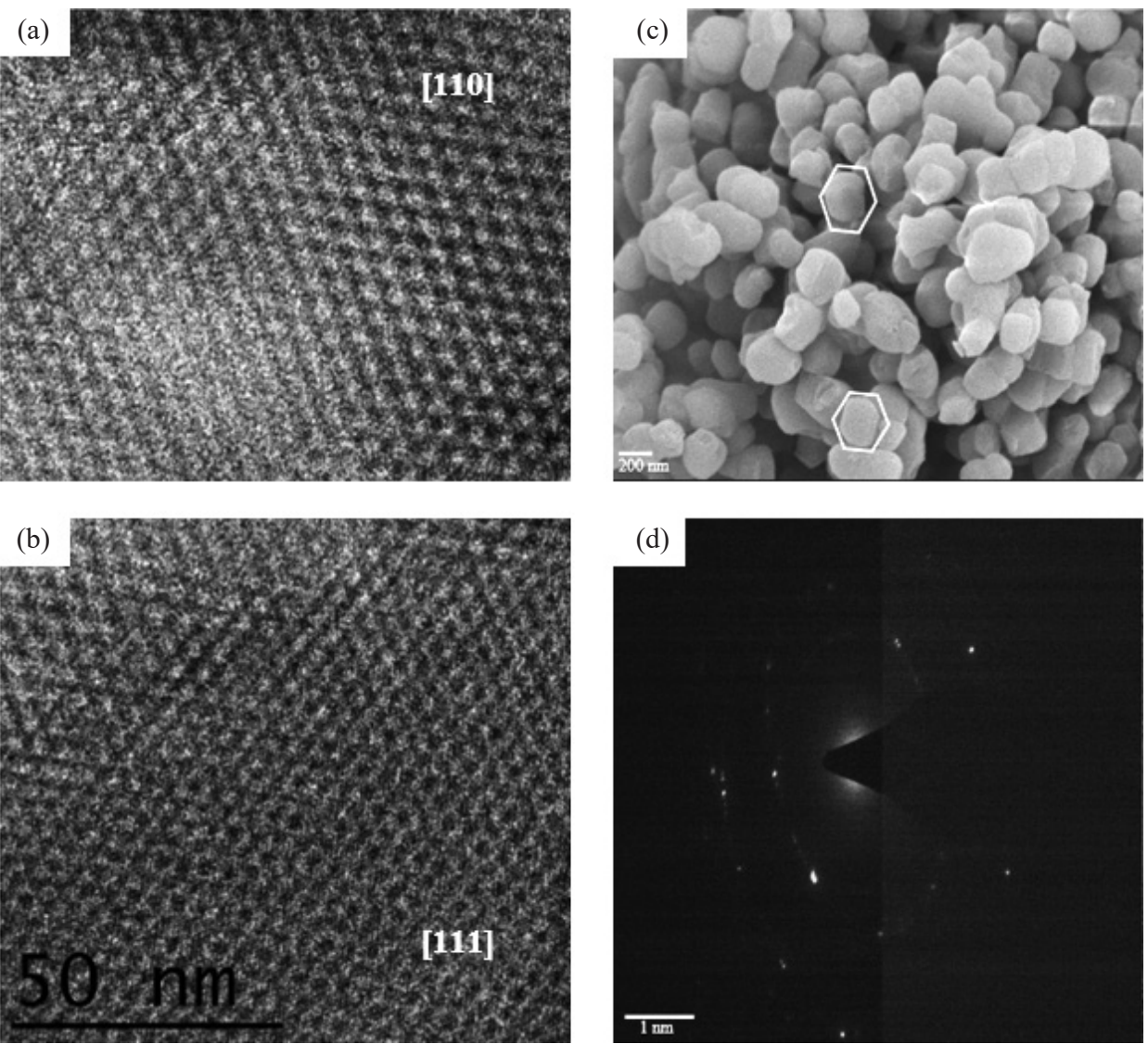

Figure 14: Morphology of MCM-48 tailored at $3000 \mathrm{rpm}$ (ethanol washed), for S10 viewed via HRTEM show (a) pore geometry from axis [110] and [111], (b) rhombohedral crystal surface, and (c) SAED image from plane [111].

\subsubsection{Surface area and porosity (SAP) analysis of tailored MCM-48}

The presence of silanol group density, $\mathrm{Si}-\mathrm{OH}$ on mesoporous silica wall and $\mathrm{N}_{2}$ adsorption-desorption isotherm of calcined MCM-48 were determined from SAP analysis via BET method. The specific surface area, pore diameter and pore volume were calculated using Barrett, Joyner and Halenda $(\mathrm{BJH})$ desorption method. Referring to Figure 15, BET surface analysis revealed that $\mathrm{N}_{2}$ isotherm pattern follows Type IV isotherm, indicating strong sorbate-adsorbate interaction in mesoporous solid, specifically for MCM- $n, n=41,48 .{ }^{33,34}$ It showed that hysteresis peaks occurred at relative pressure of $0.11-0.42\left(\mathrm{p} / \mathrm{p}^{\circ}\right)$ due to multilayer adsorption followed by capillary condensation in mesopores. BJH pore analysis revealed an average pore diameter and pore volume of $2.79 \mathrm{~nm}$ and $0.545 \mathrm{~cm}^{3} \mathrm{~g}^{-1}$, respectively. 


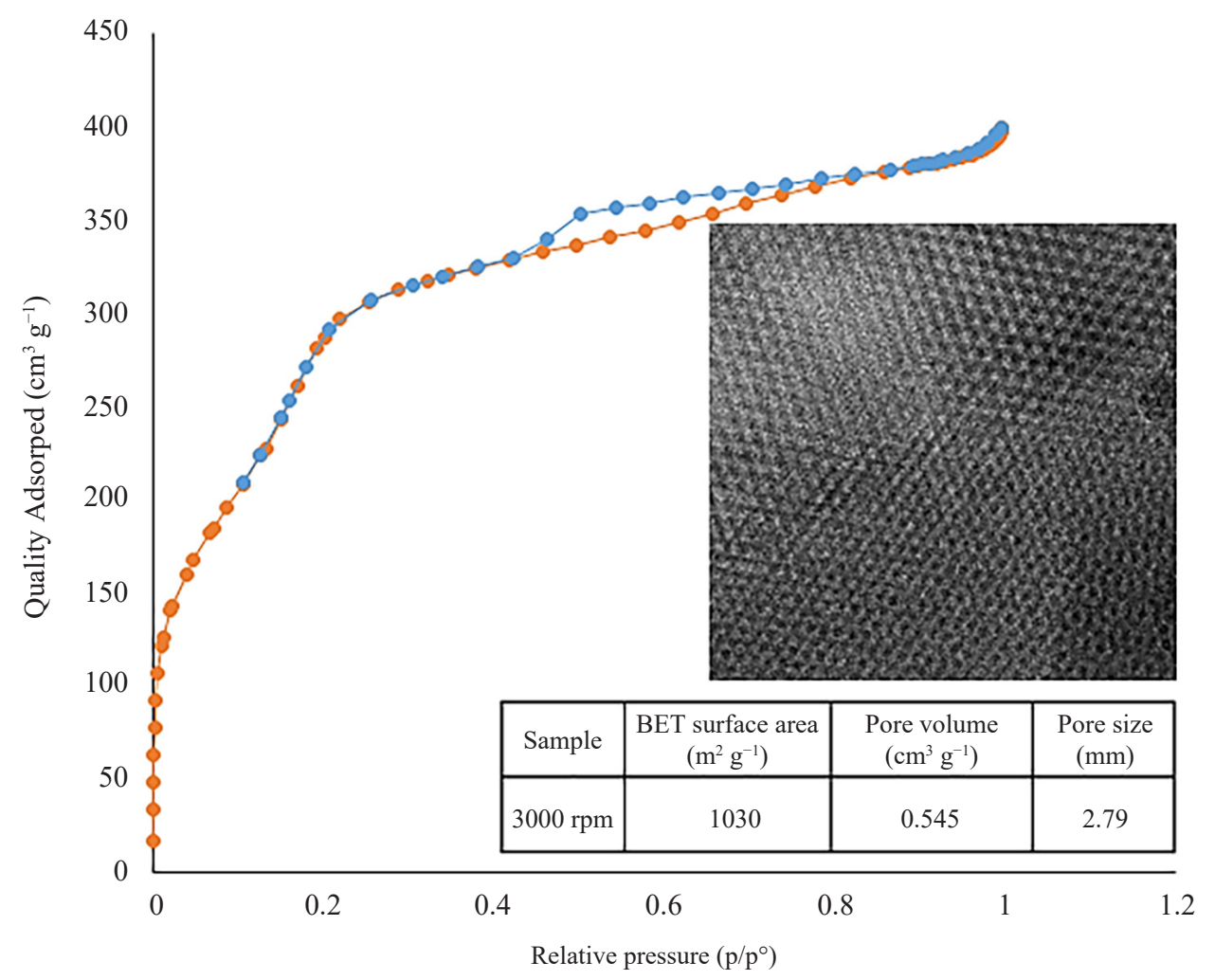

Figure 15: Surface area and porosity analysis of calcined MCM-48 silica at $3000 \mathrm{rpm}$, S10.

\subsubsection{Effect of washing medium polarity on MCM-48 agglomeration degree and particle size distribution}

The effect of washing solution (i.e., water, ethanol and methanol) on tailored MCM48 were observed via FESEM as seen in Figure 16. MCM-48 particles in S12 and S13 showed a similar pattern of dispersity and surface morphology as compared to S14. It is hypothesised that silicate species polymerise better in the presence of methanol which then leads to the formation of larger silica particles. Furthermore, physical defect was also observed on the surfaces of silica particles. This may occur during washing whereby unreacted silicates present in the mother solution further polymerised and deposited on the surface of nearby particles. Therefore, methanol might not be effective in reducing agglomeration of nanosized MCM48. Since the polarity of methanol is slightly $0.01 \mathrm{D}$ higher than ethanol $(0.69 \mathrm{D})$, therefore it is suggested that solvent with $\leq 0.5 \mathrm{D}$ might be a better alternative for washing siliceous MCM-48. 

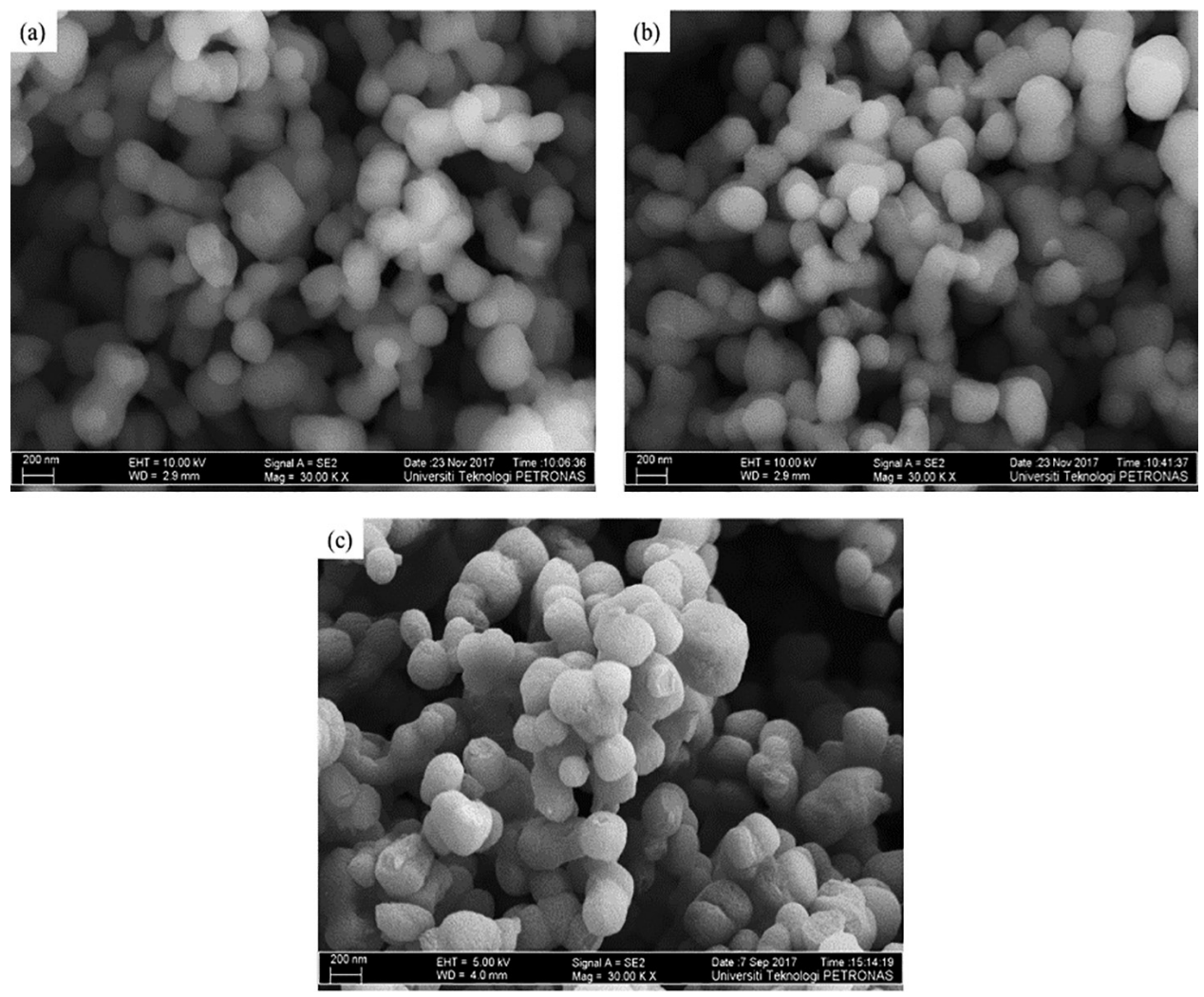

Figure 16: FESEM images of tailored MCM-48 (30K magnification) washed using (a) deionised water, (b) ethanol, and (c) methanol.

Upon further study on PSD, MCM-48 washed using deionised water and ethanol yielded the highest number of particle size within the range of 200-250 nm. Two different patterns of PSD were observed as clearly seen in Figure 17. For ethanolwashed MCM-48, it showed uniform distribution whilst water-washed MCM-48 showed bimodal distribution. It is believed that nanosized range of MCM-48 can be obtained by using water and ethanol as washing medium. 

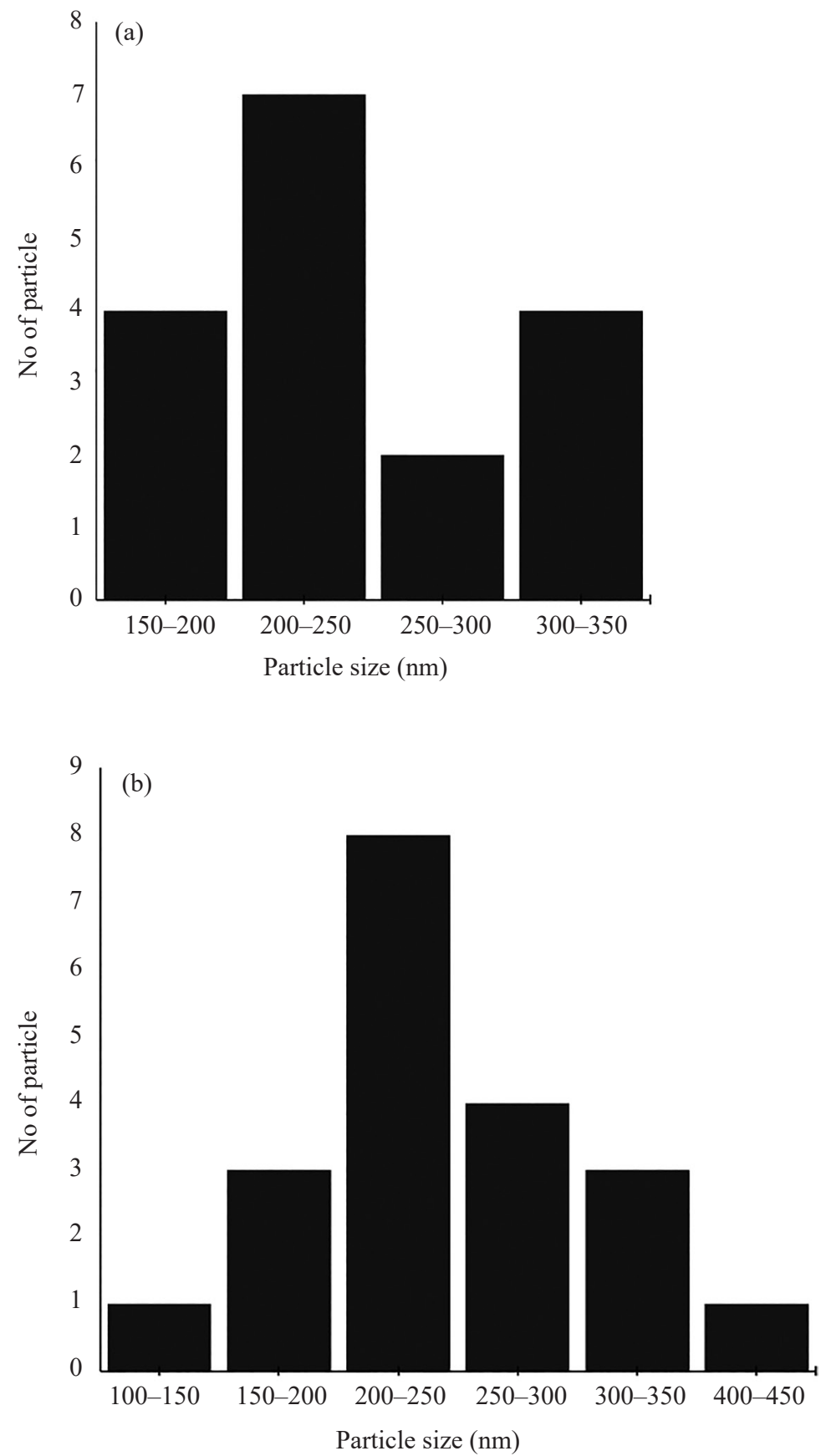

Figure 17: Particle size distribution of tailored MCM-48 washed using (a) deionised water, and (b) ethanol. 


\section{CONCLUSION}

Using a single surfactant system, nanosized MCM-48 in the range of 100-450 $\mathrm{nm}$ was successfully formed. We revealed that stirring speed from 1000-3000 rpm can provide better silica dispersion and nucleation of particles during sol-gel silica process. The stirring speed applied during silica addition has facilitated the hydrolysis and condensation reaction within a very short stirring time. Herein, small mean particle size was discovered at $1000 \mathrm{rpm}$ and $3000 \mathrm{rpm}$, ca. $200 \mathrm{~nm}$ and ca. $150 \mathrm{~nm}$ respectively. Study using different types of washing medium has shown that ethanol and deionized water are preferable than methanol for stabilising interparticle interaction, thus minimising agglomeration of hydrophilic MCM-48 silica. Furthermore, intrinsic pore properties of cubic Ia3 $d$ MCM- 48 were retained even at high stirring rate as revealed by XRD, TEM and $\mathrm{N}_{2}$ adsorption-desorption analysis. For future recommendation, kinetic of sol-gel silica process such as hydrolysis and condensation could be further controlled by tailoring the rate of silica addition and type of basic catalyst used.

\section{ACKNOWLEDGEMENTS}

This research work was supported by Yayasan Universiti Teknologi PETRONAS (YUTP-FRG grant No. 0153AA-E08), Fundamental Research Grant Scheme (FRGS grant no. FRGS/1/2018/TK02/UTP/02/3) and $\mathrm{CO}_{2}$ Research Centre (CO2RES).

\section{REFERENCES}

1. Halamová, D. et al. (2010). Naproxen drug delivery using periodic mesoporous silica SBA-15. Appl. Surf. Sci., 256, 6489-6494, https://doi.org/doi_10.1016/j. apsusc.2010.04.044.

2. Maheswari, R. et al. (2014). Synthesis, characterization, and epoxidation activity of tungsten-incorporated SBA-16 (W-SBA-16). Ind. Eng. Chem. Res., 53, 1883318839, https://doi.org/10.1021/ie501784c.

3. Kishor, R. \& Ghoshal, A. K. (2015). APTES grafted ordered mesoporous silica KIT-6 for $\mathrm{CO}_{2}$ adsorption. Chem. Eng. J., 262, 882-890, https://doi.org/10.1016/j. cej.2014.10.039.

4. Coasne, B. et al. (2006). Gas adsorption in mesoporous micelle-templated silicas: MCM-41, MCM-48, and SBA-15. Langmuir, 22, 11097-11105, https://doi.org/ $10.1021 / \mathrm{la} 061728 \mathrm{~h}$.

5. Changbum, J. et al. (2009) Syntheses of high quality KIT-6 and SBA-15 mesoporous silicas using low-cost water glass, through rapid quenching of silicate structure in acidic solution. Microp. Mesop. Mater., 124, 45-51, https://doi.org/10.1016/J. MICROMESO.2009.04.037. 
6. Roth, W. J. (2009). Facile synthesis of the cubic mesoporous material MCM-48: Detailed study of accompanying phase transformations. Adsorp., 15, 221-226. https://doi.org/10.1007/s10450-009-9161-1.

7. Monnier, A. et al. (1993). Cooperative formation of inorganic-organic interfaces in the synthesis of silicate mesostructures. Sci., 80(261), 1299-1303, https://doi. org/10.1126/science.261.5126.1299.

8. Vartuli, J. et al. (1998). The synthesis and properties of M41S and related mesoporous materials. Synth. (Stuttg)., 1, 97-119, https://doi.org/10.1007/3-54069615-6 4.

9. Firouzi, A. et al. (1997). Alkaline lyotropic silicate-surfactant liquid crystals. J. Am. Chem. Soc., 119, 3596-3610, https://doi.org/10.1021/ja963007i.

10. Narayan, K. R. et al. (1996). Template-based approaches to the preparation of amorphous, nanoporous silicas. Chem. Mater., 8(8), 1682-1701, https://doi. org/10.1021/CM960138.

11. Qisheng, H., Margolese, D. I. \& Stucky, G. D. (1996). Surfactant control of phases in the synthesis of mesoporous silica-based materials. Chem. Mater., 8(5), $1147-$ 1160, https://doi.org/10.1021/CM960137H.

12. Kumar, D. et al. (2001). MCM-41, MCM-48 and related mesoporous adsorbents: Their synthesis and characterisation. Coll. Surf. A Physicochem. Eng. Asp., 187188, 109-116, https://doi.org/10.1016/S0927-7757(01)00638-0.

13. Petitto, C. et al. (2005). Synthesis of discrete micrometer-sized spherical particles of MCM-48. Chem. Mater., 17, 2120-2130, https://doi.org/10.1021/cm050068j.

14. Díaz, I., Alfredsson, V.\& Sakamoto, Y. (2006). Transmission electron microscopy in formation and growth of ordered mesoporous materials. Curr. Opin. Coll. Interf. Sci., 11, 302-307, https://doi.org/doi_10.1016/j.cocis.2006.09.007.

15. Rahman, I. A. et al. (2007). An optimized sol-gel synthesis of stable primary equivalent silica particles. Coll. Surf. A Physicochem. Eng. Asp., 294, 102-110, https://doi.org/10.1016/j.colsurfa.2006.08.001.

16. Schumacher, K., Grün, M. \& Unger, K. (1999). Novel synthesis of spherical MCM-48. Microp. Mesop. Mater., 27, 201-206, https://doi.org/10.1016/s13871811(98)00254-6.

17. Jomekian, A. et al. (2011). Fabrication or preparation and characterization of new modified MCM-41/PSf nanocomposite membrane coated by PDMS. Sep. Purif. Technol., 80, 556-565, https://doi.org/10.1016/J.SEPPUR.2011.06.011.

18. Meléndez-Ortiz, H. I. et al. (2014). Functionalization with amine-containing organosilane of mesoporous silica MCM-41 and MCM-48 obtained at room temperature. Ceram. Int., 40(7), 9701-9707, https://doi.org/10.1016/j. ceramint.2014.02.051.

19. Meléndez-Ortiz, H. I. et al. (2014). Influence of the reaction conditions on the thermal stability of mesoporous MCM-48 silica obtained at room temperature. Ceram. Int., 40, 4155-4161, https://doi.org/10.1016/j.ceramint.2013.08.072.

20. Jomekian, A. et al. (2011). Gas transport behavior of DMDCS modified MCM-48/ polysulfone mixed matrix membrane coated by PDMS. Kor. J. Chem. Eng., 28, 2069-2075, https://doi.org/10.1007/s11814-011-0075-8. 
21. Kim, T.-W., Chung, P.-W. \& Lin, V. S.-Y. (2010). Facile synthesis of monodisperse spherical MCM-48 mesoporous silica nanoparticles with controlled particle size. Chem. Mater., 22, 5093-5104, https://doi.org/doi_10.1021/cm1017344.

22. Wei, F. Y. et al. (2010). Synthesis of mesoporous MCM-48 using fumed silica and mixed surfactants. Microp. Mesop. Mater., 131, 224-229, https://doi.org/10.1016/j. micromeso.2009.12.027.

23. Banerjee, S. et al. (2010). Pd-MCM-48: A novel recyclable heterogeneous catalyst for chemo- and regioselective hydrogenation of olefins and coupling reactions. Org. Biomol. Chem., 8, 4316, https://doi.org/doi_10.1039/c0ob00183j.

24. Boote, B., Subramanian, H. \& Ranjit, K. T. (2007). Rapid and facile synthesis of siliceous MCM-48 mesoporous materials. Chem. Commun., 4543-4545. https:// doi.org/10.1039/b706633c.

25. Tae, W. K. et al. (2004). Tailoring the pore structure of SBA-16 silica molecular sieve through the use of copolymer blends and control of synthesis temperature and time. J. Phys. Chem. B, 108(31), 11480-11489, https://doi.org/10.1021/jp048582k.

26. Chen, P.-K. et al. (2013). New synthesis of MCM-48 nanospheres and facile replication to mesoporous platinum nanospheres as highly active electrocatalysts for the oxygen reduction reaction. Chem. Mater., 25, 4269-4277, https://doi. org/10.1021/cm402349f.

27. Díaz, I., Pérez-Pariente, J. \& Terasaki, O. (2004). Structural study by transmission and scanning electron microscopy of the time-dependent structural change in M41S mesoporous silica (MCM-41 to MCM-48, and MCM-50). J. Mater. Chem., 14, 48-53, https://doi.org/10.1039/B310281E.

28. Zhai, S. R. et al. (2002). The synthesis of mesoporous molecular sieve MCM-48 using two surfactants as co-template. Acta Phys. Chim. Sin., 18, 911-915, https:// doi.org/10.3866/PKU.WHXB20021009.

29. Zhao, W. et al. (2010). Synthesis of high quality MCM-48 with binary cationicnonionic surfactants. Langmuir, 26, 6982-6988, https://doi.org/10.1021/ la9042155.

30. Kaskel, S. (2008). Characterisation of porous solids VIII. Paper presented at the Proceedings of the 8th International Symposium on the Characterisation of Porous Solids, 10-13 June, Edinburgh.

31. Rouquerol, J. et al. (1994). Recommendations for the characterization of porous solids (technical report). Pure Appl. Chem., 66, 1739-1758, https://doi. org/10.1351/pac199466081739

32. Alfredsson, V. \& Anderson, M. W. (1996). Structure of MCM-48 revealed by transmission electron microscopy. Chem. Mater., 8, 1141-1146, https://doi. org/10.1021/cm950568k.

33. Schumacher, K. et al. (2000). Characterization of MCM-48 Materials. Langmuir, 16, 4648-4654, https://doi.org/10.1021/la991595i.

34. Sang, W. Y. \& Ching, O. P. (2017). Tailoring MCM-41 mesoporous silica particles through modified sol-gel process for gas separation. Paper presented at the in AIP Conference Proceedings 1891, 3-5 April, Kedah, Malaysia, https://doi. org/10.1063/1.5005480. 